\title{
DIMENSION THEORY IN COMPLETE ORTHOCOMPLEMENTED WEAKLY MODULAR LATTICES
}

\author{
BY \\ ARLAN RAMSAY $\left.{ }^{1}\right)$
}

1. Introduction. In [9] L. H. Loomis studies an abstract version of the von Neumann-Murray dimension theory for rings of operators in Hilbert space. He takes as context a complete orthocomplemented weakly modular (oc.w.m.) lattice $L$ together with a dimensional equivalence relation (d.e.r.) on $L$, i.e., an equivalence relation on $L$ satisfying axioms $\mathrm{A}, \mathrm{B}, \mathrm{C}$ and $\mathrm{D}^{\prime}$ $[9$, p. 4]. (See $\$ 2$ for this and other definitions and background material.) Hence it seems of interest, particularly from the lattice theoretic point of view, to have necessary and sufficient conditions on a complete oc.w.m. lattice $L$ in order that there exist a d.e.r. on $L$. The answer to this particular question is fairly simple, and is given in Theorem 8.2 of this paper: If $L$ is a complete oc.w.m. lattice, then there exists a d.e.r. on $L$ if and only if $L$ satisfies the following standard covering condition [2, p. 100]: (p) if $a$ covers $a \wedge b$ then $a \bigvee b$ covers $b$. Of course dimension theory ordinarily concentrates on the locally finite case. This case is considered in Theorem 4.24: If $L$ is a complete oc.w.m. lattice, then there exists a locally finite d.e.r. on $L$ if and only if $L$ is locally modular. Related results of purely lattice theoretic interest are that if $L$ is a locally modular complete oc.w.m. lattice then $\{x \in L:[0, x]$ is modular $\}$ is the largest modular ideal in $L$, and the relation of being a modular pair is symmetric in $L$ (Theorems 4.17 and 5.4).

A lattice will be called a von Neumann lattice if it is complete, complemented, and modular, and the lattice operations are continuous, i.e., if axioms I, II, III, IV and V introduced by John von Neumann in [18] are satisfied. The theory of perspectivity in von Neumann lattices is developed extensively in [14] and [18], and our results in $\$ \$ 4,5$ and 6 are an application and in a certain sense an extension of that theory. In particular we call attention to the fact that some of the harder results on perspectivity imply that if $L$ is an oc. von Neumann lattice then perspectivity is a d.e.r. on $L$. Some of the facts in [14] were first proved by T. Iwamura in [7], for instance complete additivity of the dimension function. However we will refer to [14] since it is a complete and unified exposition.

$\$ 2$ of the paper contains background material and some basic lemmas

Presented to the Society August 30, 1963 under the title Dimension theory in an arbitrary complete orthomodular lattice; received by the editors March 3, 1964.

$\left({ }^{1}\right)$ The work on this paper was partly supported by the Army Research Office (Durham). 
and definitions. $\S 3$ then presents an intrinsic decomposition of the general complete oc.w.m. lattice into four parts. This provides the context for the rest of the paper, and a more detailed outline is given at the end of $\$ 3$.

Many of the results in this paper were contained in the author's thesis (Harvard, 1962). The author is happy to express his gratitude to his thesis advisor Professor L. H. Loomis for his guidance and encouragement. The author is also especially indebted to Professor G. Birkhoff and Professor G. W. Mackey for helpful advice and criticism.

2. Preliminaries. For a lattice which has them, least and greatest elements will almost always be denoted by 0 and 1 . A lattice is orthocomplemented (oc.) if it has least and greatest elements and an involutory anti-automorphism $x \rightarrow x^{\prime}$ such that for each $x, x^{\prime}$ is a complement of $x\left(x \vee x^{\prime}=1, x \wedge x^{\prime}=0\right)$ $\left[9\right.$, p. 3]. The mapping $x \rightarrow x^{\prime}$ is the orthocomplementation. Elements $x, y$ of an oc. lattice are orthogonal, written $x \perp y$, if and only if $x \leqq y^{\prime}$. A set $S$ in an oc. lattice is orthogonal if and only if $x \perp y$ whenever $x$ and $y$ are distinct elements of $S$, and an indexed set $\left\{x_{i}: i \in I\right\}$ is orthogonal if $i \neq j$, $i \in I$ and $j \in I$ implies $x_{i} \perp x_{j}$.

The least upper bound of a set $S$ will be denoted by $\sup S$ and the greatest lower bound by inf $S$, when they exist, except that the least upper bound of an orthogonal set may be denoted by $\Sigma S(x=\Sigma S$ means that $S$ is an orthogonal set with least upper bound $x$ ). In the same way, $x+y$ may be used for $x \vee y$ if $x \perp y$, and $x-y$ for $x \wedge y^{\prime}$ if $x \geqq y$ (to write $x+y$ will imply that $x \perp y$ ).

An oc. lattice is weakly modular (w.m.) if and only if $[9$, p. 4]:

$$
x \leqq y \text { implies } y=x+(y-x) \quad\left(=x \bigvee\left(y \wedge x^{\prime}\right)\right)
$$

Lemma 3 of [9] gives several conditions equivalent to weak modularity, one of which is clearly equivalent, just by orthocomplementation:

$$
x \geqq y \text { implies } y=x \wedge\left(y \vee x^{\prime}\right) \text {. }
$$

For the remainder of this section we restrict our attention to a complete oc.w.m. lattice $L$. Then by $M_{3}, a \geqq x, b>y$ and $a \perp b$ imply that $a+b$ $>x+y$.

Lemma 2.1. Let $T \subseteq L$ be an orthogonal set, let $M=I I\{[0, t]: t \in T\}$, and define $\alpha: M \rightarrow[0, \Sigma T]$ by setting $\alpha(f)=\sup \{f(t): t \in T\}$ for $f$ in $M$. Then $\alpha$ is one-one and preserves arbitrary joins and meets, and if $f$ and $g$ are in $M$ with $g(t)=t-f(t)$ for $t \in T$, then $\alpha(g)=(\Sigma T)-\alpha(f)$.

Proof. If $S \subseteq M$, then $(\sup S)(t)=\sup \{f(t): f \in S\}$ for $t \in T$, so $\alpha(\sup S)$ $=\sup \{\alpha(f): f \in S\}$ since taking joins is completely commutative. Now if $f$ and $g$ are in $M$ and $g(t)=t-f(t)$ for $t \in T$, then $f\left(t_{1}\right) \perp g\left(t_{2}\right)$ for any $t_{1}$ and $t_{2}$ in $T$, so $\alpha(f) \perp \alpha(g)$. But $\alpha(f \bigvee g)=\Sigma T$, so $\alpha(g)=(\Sigma T)-\alpha(f)$, 
by the above-mentioned consequence of weak modularity. By taking orthocoirplements in $[0, \Sigma T]$, it follows that $\alpha$ also preserves arbitrary meets. To prove (one-one)-ness, let $f \in M, g \in M$ and $f \neq g$; then we may suppose, by symmetry, that there is a $t_{0} \in T$ with $f\left(t_{0}\right) \neq g\left(t_{0}\right)$. Setting $h=f \bigvee g$, it follows that $h\left(t_{0}\right)>g\left(t_{0}\right)$ and $\sup \left\{h(t): t_{0} \neq t \in T\right\} \geqq \sup \left\{g(t): t_{0} \neq t \in T\right\}$. Using the same consequence of weak modularity as before, it follows that $\alpha(f) \bigvee \alpha(g)=\alpha(h)>\alpha(g)$, so $\alpha(f) \neq \alpha(g)$. Q.E.D.

REMARK. This lemma generalizes a special case of the remark on p. 489 of $[1]$, and is also suggested by the results on p. 73 of [2]. For complete oc. lattices the statement is equivalent to weak modularity, since by applying it to the case $T=\left\{x, x^{\prime}\right\}$ the property $\left(\mathrm{M}_{3}\right)$ can be derived. It also has Lemmas 28 and 29 on p. 536 of [8] and Lemma 5 on p. 6 of [9] as immediate corollaries.

A relation $\sim$ on $L$ will be called a dimensional equivalence relation (d.e.r.) if it is an equivalence relation and satisfies the following axioms $[9$, p. 4]:

(A) $a \sim 0$ implies $a=0$.

(B) If $b \sim a_{1}+a_{2}$ then there exist $b_{1}$ and $b_{2}$ such that $b_{1} \sim a_{1}, b_{2} \sim a_{2}$ and $b=b_{1}+b_{2}$.

(C) If $\left\{a_{i}: i \in I\right\}$ and $\left\{b_{i}: i \in I\right\}$ are orthogonal, and $a_{i} \sim b_{i}$ for every $i$, then $\Sigma\left\{a_{i}: i \in I\right\} \sim \Sigma\left\{b_{i}: i \in I\right\}$.

$\left(D^{\prime}\right)$ If $a$ and $b$ are perspective then $a \sim b$.

(Elements $a$ and $b$ are perspective if they have a common complement, i.e., there is a $c$ with $a \vee c=b \vee c=1, a \wedge c=b \wedge c=0$.) If $\sim$ is a d.e.r. on $L$ then $(L, \sim)$ is a dimension lattice. If $\sim$ is a d.e.r. on $L$ an element $x \in L$ is called finite (with respect to $\sim$ ) if $x \sim y \leqq x$ implies $y=x$, and otherwise $x$ is infinite $[9$, p. 6]. If every nonzero element of $L$ is infinite then both $\sim$ and $(L, \sim)$ are said to be of type III $[9$, p. 2$]$. We shall call both $\sim$ and $(L, \sim)$ locally finite if every nonzero element dominates a nonzero finite element. Let $F$ be the set of all finite elements in a dimension lattice. Then Lemma $6[9$, p. 7$]$ and Theorem $7[9$, p. 25] together say that $F$ is an ideal; if $(L, \sim)$ is locally finite then it is also true that every element of $L$ is a join of elements of $F$. If $a<b$, and $c$ is such that $a \bigvee c=d_{1}=b \bigvee c$ and $a \wedge c=d_{0}=b \wedge c$, then by the corollary to Lemma 41 of $[9$, p. 24], we have $b-d_{0} \sim d_{1}-c \sim a-d_{0}<b-d_{0}$ so $b-d_{0}$ is infinite, hence $d_{1}$ is infinite and is not in $F$. Thus $x \in F$ implies $[0, x]$ has no five element nonmodular sublattice, and hence is modular. Thus $F$ is modular as a sublattice of $L$.

If $L$ is any complete oc.w.m. lattice we shall call a sublattice $M$ closed if it is closed under arbitrary joins and meets: $S \subseteq M$ implies $\sup S \in M$ and inf $S \in M$. The center of $L$ is the set of all $x$ such that the isomorphism of $[0, x] \times\left[0, x^{\prime}\right]$ into $L$ given by Lemma 2.1 is onto. We refer to [15] for properties of the center of $L$; the proofs given there apply to complete oc.w.m. lattices. In particular, an element is central if and only if it has 
a unique complement, the center is a closed sublattice of $L$, and $(\sup S) \wedge b$ $=\sup \{x \wedge b: x \in S\}$ if $S$ is a subset of the center or if $b$ is in the center. Thus the center is a complete Boolean algebra, and if $T$ is an orthogonal subset of the center with $\sup T=1$, the mapping in Lemma 2.1 is an isomorphism onto $L$. For $e$ in the center of $L$ and $x$ in $L, x=(x \wedge e) \vee\left(x \wedge e^{\prime}\right)$, so $x \wedge e=0$ implies $x \leqq e^{\prime}$. Weak modularity shows that this last property characterizes central elements of $L$.

If $\left(L_{1}, \sim_{1}\right)$ and $\left(L_{2}, \sim_{2}\right)$ are dimension lattices they have a direct product $(L, \sim)$ formed by setting $L=L_{1} \times L_{2}$ and defining $\left(x_{1}, x_{2}\right) \sim\left(y_{1}, y_{2}\right)$ to mean $x_{1} \sim_{1} y_{1}$ and $x_{2} \sim_{2} y_{2}$. As elements of the center are related to lattice decompositions, so certain elements of the lattice are related to dimension lattice decompositions: an element $e$ of a dimension lattice $(L, \sim)$ is invariant if $x \sim y \leqq e$ implies $x \leqq e\left[9\right.$, Lemma 21, p. 12]. If we define $\sim_{e}$ to be $\sim$ restricted to $[0, e]$, invariance of $e$ is exactly the condition that makes $\left([0, e], \sim_{e}\right)$ a dimension lattice direct factor of $(L, \sim)[9$, Theorem 2, p. 13]. Also the set of invariant elements is a closed Boolean subalgebra of the center of $L[9$, Theorem 2]. Every dimension lattice is uniquely a direct product of a locally finite dimension lattice and a type III dimension lattice [9, pp. $2 ; 19]$.

If $E$ is any closed Boolean subalgebra of a complete oc.w.m. lattice $L$, and $x \in L$ write $|x|$ for the least element of $E$ greater than or equal to $x$; $|x|$ will be called the $E$-cover, or the central cover in case $E$ is the center of $L$. (See $[18$, p. 242] or $[9$, p. 13].) If $E$ is the set of invariant elements of a dimension lattice, Loomis calls $|x|$ the hull of $x[9$, p. 13]. Notice that for $T \subseteq L,|\sup T|=\sup \{|t|: t \in T\}$. Another useful fact is contained in the following lemma.

Lемма 2.2. If $x$ and $y$ are perspective elements of $L$, then $|x|=|y|$.

Proof. (The proof for von Neumann lattices given on p. 243 of [18] applies, but we shall give a slight variation which uses the orthocomplementation.) Let $z$ be a common complement of $x$ and $y$. Then $x \wedge z=0$ and $z \geqq\left|z^{\prime}\right|^{\prime}$, so $x \wedge\left|z^{\prime}\right|^{\prime}=0$; hence $x \leqq\left|z^{\prime}\right|$ and $|x| \leqq\left|z^{\prime}\right|$. But $x^{\prime} \wedge z^{\prime}$ $=0$, so $\left|z^{\prime}\right| \leqq|x|$ in the same way. Thus $|x|=\left|z^{\prime}\right|$, and the same proof shows that $|y|=\left|z^{\prime}\right|$, so $|x|=|y|$. Q.E.D.

Lemma 2.3. If $T$ is a subset of $L$ such that distinct elements of $T$ have orthogonal E-covers, then the natural isomorphism of $\mathrm{II}\{[0,|t|]: t \in T\}$ onto $[0,|\Sigma T|]$ takes $\coprod\{\mid 0, t]: t \in T\}$ onto $[0, \Sigma T]$.

Proof. Clear.

Let $L$ be a complete oc.w.m. lattice and $E$ a closed subalgebra of the center. Some of the interaction between $E$ and $L$ can be studied via the functions $\lambda_{a}:[0,|a|] \cap E \rightarrow[0, a]$, defined for $a \in L, e \leqq|a|, e \in E$ by $\lambda_{a}(e)=a \wedge e$. 
Lemma 2.4. For any $a \in L, \lambda_{a}$ is a Boolean algebra isomorphism of $[0,|a|]$ $\neg F$. into the center of $[0, a]$.

Proof. By weak modularity, $|a \wedge e|=e$, so $\lambda_{a}$ is one-one. Clearly $\lambda_{a}\left(e_{1} \wedge e_{2}\right)=\lambda_{a}\left(e_{1}\right) \wedge \lambda_{a}\left(e_{2}\right)$, and $\lambda_{a}\left(e_{1} \vee e_{2}\right)=\lambda_{a}\left(e_{1}\right) \bigvee \lambda_{a}\left(e_{2}\right)$ because $e_{1}$ and $e_{2}$ are in the center of $L$. Thus if $e \leqq|a|$ is in $E$, then $\lambda_{a}(|a|-e)$ is a complement of $\lambda_{a}(e)$ in $[0, a]$. Furthermore, if $e \leqq|a|$ is in $E$ and $x \leqq a$, then $x \wedge \lambda_{a}(e)=x \wedge a \wedge e=x \wedge e$. Hence $x \wedge \lambda_{a}(e)=0$ implies $x \leqq e^{\prime}$, so $x \leqq|a|$ $-e$ and $x \leqq \lambda_{a}(|a|-e)$. Thus $\lambda_{a}(|a|-e)$ is the only complement of $\lambda_{a}(e)$ in $[0, a]$. Q.E.D.

Thus if $x$ is an atom in $L$, then $|x|$ is an atom in $E$.

DEFINITION 2.5. An element $a \in L$ is called multiplicity free with respect to $E$ if $\lambda_{a}$ takes $[0,|a|] \cap E$ onto $[0, a]$.

If $E$ is the center of $L$, the qualifying phrase "with respect to $E$ " may be omitted. In a dimension lattice $(L, \sim)$ an element is called simple relative to $\sim$ if and only if it is multiplicity free with respect to the Boolean algebra of invariant elements $[9$, p. 2]. The terminology we use is borrowed from the theory of group representations [11]. The following lemma helps to clarify the nature of multiplicity free elements.

LEMma 2.6. The following conditions are equivalent:

(i) $a$ is multiplicity free with respect to $E$,

(ii) the mapping $x \rightarrow|x|$ from $[0, a]$ into $[0,|a|] \cap E$ is one-one,

(iii) if $x \leqq a, y \leqq a$ and $x \perp y$, then $|x| \wedge|y|=0$.

Proof. Take $\lambda_{a}$ as in Definition 2.5 and let $\psi_{a}$ be the mapping $x \rightarrow|x|$ of condition (ii). Then $a$ is multiplicity free if and only if $\lambda_{a} \circ \psi_{a}$ is the identity on $[0, a]$. But $\psi_{a} \circ \lambda_{a}$ is always the identity on $[0,|a|] \cap E$, so $\lambda_{a} \circ \psi_{a}$ is the identity if and only if $\psi_{a}$ is one-one. Thus (i) and (ii) are equivalent.

If $\psi_{a}$ is one-one and $x, y$ are elements of $[0, a]$ with $e=|x| \wedge|y| \neq 0$, then $x \wedge e=y \wedge e$ because $|x \wedge e|=e=|y \wedge e|$. Hence $|x| \wedge|y| \neq 0$ implies $x \not \perp y$. Thus (iii) follows from (ii). On the other hand suppose (iii) holds and let $|x|=\psi_{a}(x)=\psi_{a}(y)=|y|$. Then $|x \vee y|=|x| \bigvee|y|=|x|$. Set $z=(x \vee y)-x$. Then $|z| \leqq|x \vee y|=|x|$, and $x \perp z$, so $|z|=|x| \wedge|z|=0$. Hence $z=0$, so $x \geqq y$. Similarly $y \geqq x$, so $x=y$. Thus $\psi_{a}$ is one-one, and (iii) implies (ii). Q.E.D.

Another helpful fact is this:

LEMMA 2.7. Let $L$ be a complete oc.w.m. lattice and let $E_{1}$ and $E_{2}$ be closed Boolean subalgebras of the center of $L$ with $E_{1} \subseteq E_{2}$. If $a$ is multiplicity free with respect to $E_{1}$ then $a$ is multiplicity free with respect to $E_{2}$.

Proof. This is obvious from Definition 2.5.

3. A decomposition. We begin with a definition. The term "join-dense" was suggested by M. D. Maclaren, and the motivation for studying local 
modularity arises from the fact that locally finite dimension lattices are locally modular as lattices $(\$ 2)$.

Definition 3.1. Let $L$ be a complete oc.w.m. lattice. A set $X \subseteq L$ will be called join-dense in $L$ if and only if $a \in L$ implies $a=\sup (X \cap[0, a])$. An ideal of $L$ will be called modular if it is modular as a sublattice of $L$. Call $L$ locally modular if and only if $L$ has a join-dense modular ideal.

Now let $L$ be an arbitrary complete oc.w.m. lattice with center $E$. Let $e_{1}=\sup S_{1}$ where $S_{1}$ is maximal among the orthogonal subsets of $\{e \in E:[0, e]$ is locally modular $\}$, and set $L_{1}=\left[0, e_{1}\right]$. If whenever $e \in S_{1}$ we have chosen $M_{e}$ to be a join-dense modular ideal in $[0, e]$, then the natural isomorphism of $\prod\left\{[0, d]: e \in S_{1}\right\}$ onto $L_{1}$ takes $\prod\left\{M_{e}: e \in S_{1}\right\}$ onto a join-dence modular ideal in $L_{1}$. Hence $L_{1}$ is locally modular. The maximality of $S_{1}$ implies that $L_{1}$ is the largest locally modular direct summand of $L$. Let $e_{2}=\sup S_{2}$, where $S_{2}$ is maximal among the orthogonal subsets of $\left\{e \in E: e \perp e_{1}\right.$ and $[0, e]$ contains no nonzero multiplicity free elements $\}$. Then $L_{2}=\left[0, e_{2}\right]$ is the largest direct summand of $L$ with these two properties: (a) $L_{2}$ has no nonzero locally modular direct summand, (b) $L_{2}$ contains no nonzero multiplicity free elements. A third application of Zorn's Lemma enables us to take a largest atom-free direct summand of $\left[0,\left(e_{1} \bigvee e_{2}\right)^{\prime}\right]$, call it $L_{3}=\left[0, e_{3}\right]$. Then every nonzero central element of $L_{3}$ dominates a nonzero multiplicity free element, by choice of $L_{2}$, so there is a set $X$ of multiplicity free elements of $L_{3}$ such that $x_{1} \neq x_{2}$ in $X$ implies $\left|x_{1}\right| \perp\left|x_{2}\right|$, and $\Sigma\{|x|: x \in X\}$ $=e_{3}$. Then $\Sigma X$ is a multiplicity free element with central cover $e_{3}$. In fact, $e_{3}$ is the largest element of the set of central elements $e$ such that $e$ is the central cover of a multiplicity free element and $[0, e]$ has no nonzero locally modular direct summand. Lastly, $L_{4} 2\left[0,\left(e_{1} \bigvee e_{2} \bigvee e_{3}\right)^{\prime}\right]$ has no nonzero locally modular direct summand and every nonzero central element of $L_{4}$ dominates an atom; in fact $L_{4}$ is the largest such direct summand of $L$.

In the rest of the paper, $\$ 4$ deals with $L_{1}$, showing that a "nice" dimension theory is implicit in the local modularity, $\$ 5$ shows that $L_{1}$ is semi-modular, $\$ 6$ gives sufficient conditions that $L=L_{1}$ and necessary and sufficient conditions that $L_{4}=\{0\}$ and $L_{3} \times L_{4}=\{0\}, \S 7$ shows that $L_{2} \times L_{3}$ is essentially type III, $\S 8$ returns to the general $L$, and $\$ 9$ gives examples of $L_{3} \neq\{0\}$ and $L_{4} \neq\{0\}$.

4. The locally modular case. Throughout this section, $L$ will denote a complete oc.w.m. lattice which is locally modular, and $E$ will denote the center of $L$. The union of a chain of modular ideals is a modular ideal, and a set containing a join-dense set is join-dense, so by Zorn's Lemma there is an ideal $J$ in $L$ which is join-dense and which is maximal in the collection of modular ideals of $L$. It will be seen in Theorem 4.18 that $J=\{a \in L:[0, a]$ is modular $\}$, and hence that $L$ has only one such ideal. However we have no short proof of this fact and hence must proceed with an apparently arbitrary choice of $J$. 
The main goal of this section is to define a locally finite d.e.r. $\sim$ on $L$ so that e ry element of $E$ is invariant (see \$2). This will be accomplished as follows: $\sim$ can be defined on $J$ using the modularity of $J$ and a "dimension function" $d$ on $J$ can be associated with $\sim$. Then extend $d$ to a function $d$ on $L$ and define $a \sim b$ to mean $d(a)=d(b)$. The appropriate properties of $d$ then show that $\sim$ is as desired.

If $a \in J$, then $[0, a]$ is modular, so $[0, a]$ is a von Neumann lattice, by a theorem of I. Kaplansky [8]. Hence in dealing with elements of $J$ the results of [14] and [18] are available. For $a$ and $b$ in $J$ define $a \sim b$ to mean $a$ and $b$ are perspective in $[0, a \bigvee b]$. By Theorem 3.1 on p. 17 of [18] $a \sim b$ if and only if $x \in J$ and $x \geqq a \vee b$ implies $a$ and $b$ are perspective in $[0, x]$, and $a \sim b$ if and only if there is an $x \in J$ such that $x \geqq a \bigvee b$ and $a$ is perspective in $b$ in $[0, x]$. From this it follows easily that $\sim$ is an equivalence relation, because perspectivity is an equivalence relation on any von Neumann lattice [18, p. 265].

Definition 4.1. Elements $a$ and $b$ of $J$ are related, written $a \rho b$, if there exist $x$ and $y$ with $0<x \leqq a, 0<y \leqq b$, and $x \sim y$. Otherwise $a$ and $b$ are unrelated, written $a \delta b$.

For $a$ and $b$ in $J$, write $a \prec b$ to mean that there is an $a_{1} \leqq b$ with $a \sim a_{1}$, not excluding the possibility that $a \sim b$. Then $a \delta b$ if and only if $x \leqq a$ and $x \prec b$ imply $x=0$. For $a \in J$ set $J_{1}(a)=\{x \in J: 0<y \leqq x$ implies $y \rho a\}$ and $J_{2}(a)=\{x \in J: x \delta a\}$. For any $x$ and $y$ in $L, y$ is a complement of both $(x \vee y)-y$ and $x-(x \wedge y)$ in $[0, x \bigvee y]$ (see $[9$, p. 24]), so if $x$ and $y$ are in $J$ and $x$ is not orthogonal to $y$, then $x_{\rho} y$. Suppose $z \sim x$ and $x_{\rho} a$, say $0<x_{1} \leqq x$ and $x_{1} \sim y_{1}$ with $0<y_{1} \leqq a$, and let $c=x \bigvee z \bigvee a$. Then $\sim$ and perspectivity are the same on $[0, c]$, and $[0, c]$ is a von Neumann lattice so the perspectivity mapping of $[0, x]$ onto $[0, z]$ takes $x_{1}$ into an element $z_{1} \leqq z$ with $z_{1} \sim x_{1}\left[18\right.$, pp. 17-19]. Thus $z_{1} \sim y_{1}$, and $z \rho a$. Hence $x \in J_{1}(a)$ and $y \in J_{2}(a)$ implies $x \perp y$. Setting $e_{1}=\sup J_{1}(a)$ and $e_{2}$ $=\sup _{2}(a)$, we have $e_{1} \perp e_{2}$.

Lemma 4.2. (See Theorem 3 on $p$. 13 of $[9]$ ) $e_{1}=|a|, e_{2}=|a|^{\prime}$.

Proof. If $x \in J$ and $x \wedge e_{2}=0$, then $0<y \leqq x$ implies $y_{\rho} a$, so $x \in J_{1}(a)$. Hence if $x \in L$ and $x \wedge e_{2}=0$, then $x$ is a join of elements of $J_{1}(a)$, so $x \leqq e_{1}$. Thus $e_{1}$ is the only complement of $e_{2}, e_{1}$ and $e_{2}$ are in $E$, and $e_{1}+e_{2}=1$. Since $a \leqq e_{1}$, this implies that $|a| \leqq e_{1}$. If $x \in J_{1}(a)$, then

$$
x=\sup \{y: y \leqq x \text { and } y \prec a\} \text {, }
$$

so $x \leqq|a|$, since $y \leqq|y| \leqq|a|$ whenever $y \prec a$ (Lemma 2.2). Hence $e_{1} \leqq|a|$.

Theorem 4.3. If $a \in J$, the function $e \rightarrow e \wedge a$ takes $[0,|a|] \cap E$ isomorphically onto the center of $[0, a]$.

Proof. It is an isomorphism into the center of $[0, a]$, by Lemma 2.4, so 
suppose $c$ is in the center of $[0, a]$. Then $y \leqq a$ and $y \prec c$ implies $y \leqq c$, so $J_{1}(c) \cap[0, a]=[0, c]$ and $J_{2}(c) \cap[0, a]=[0, a-c]$. Set $b=|c| \wedge a$. Then $b \geqq c$ and $b \in J$. Now $b \leqq|c|$, so $b \in J_{1}(c)$; but also $b \leqq a$, so $b \leqq c$. Q.E.D.

To define a dimension function on $J$, we shall use an element of $J$ with central cover 1 as a standard to which we compare the other elements (see $[9$, p. 20], $[15$, p. 228]), otherwise the resulting equivalence relation would not be locally finite. The next theorem shows that such an element exists and we then proceed with the construction of the dimension function. For $a \in L$ it will be convenient to write $J_{0}(a)$ for $J \cap[0, a]$.

Theorem 4.4. There is an element $u \in J$ with $|u|=1$.

Proof. Let $T$ be maximal among the orthogonal subsets of $J$ with the property that $t_{1} \neq t_{2}$ in $T$ implies $\left|t_{1}\right| \perp\left|t_{2}\right|$. Then $\Sigma\{|t|: t \in T\}=1$. Let $\alpha$ be the natural isomorphism of $\prod\{[0,|t|]: t \in T\}$ onto $L$. Then $\alpha\left(\prod\left\{J_{0}(|t|): t \in T\right\}\right)$ is a modular ideal in $L$ containing $J$ and hence equals $J$. Hence, if $u=\Sigma T$ then $u \in J$ and $|u|=1$.

Let $S$ denote the Stone space of $E$, i.e., the set of all maximal dual ideals of $E$. For $e \in E, \sigma(e)$ will denote the set $\{s \in S: e \in s\}$ and $\phi(e)$ will denote the characteristic function of $\sigma(e)$. Then $S$ is given the weakest topology in which all $\phi(e)$ are continuous. The result is that $\sigma$ is an isomorphism of $E$ onto the Boolean algebra of all open-and-closed subsets of $S$. We shall write $C^{*}(S)$ for the set of continuous extended real-valued functions on $S$ and $C_{*}(S)$ for the set of elements of $C^{*}(S)$ which take on the values $+\infty$ and $-\infty$ at most on a nowhere dense set. Then $C^{*}(S)^{+}\left(C_{*}(S)^{+}\right)$ will denote the set of positive valued elements of $C^{*}(S)\left(C_{*}(S)\right)$. The necessary facts about this situation are found in [4], [16] and [17]. In particular, $S$ is compact and Hausdorff, and the closure of any open set is open since $E$ is complete. Hence $C^{*}(S)$ and $C^{*}(S)^{+}$are complete lattices while $C_{*}(S)$ and $C_{*}(S)^{+}$are conditionally complete. Pointwise function operations make sense in $C^{*}(S)^{+}$if we agree that always $a+(+\infty)=+\infty$ $+a=+\infty$, and $a \cdot(+\infty)=+\infty \cdot(a)=+\infty$ if $a \neq 0$, but $0 \cdot(+\infty)$ $=(+\infty) \cdot 0=0$. To make $C_{*}(S)$ a vector lattice we need slightly different operations; to define them one needs to know another fact: if $h$ is realvalued and continuous on a residual set $R$ in $S$ then there is exactly one $h^{*}$ in $C_{*}(S)$ whose restriction to $R$ is $h$. (A set is residual if and only if its complement is first category, countable intersections of residual sets are residual, and open dense sets are residual.) Now if $f$ and $g$ are in $C_{*}(S)$, let $U$ and $V$ be open dense sets on which $f$ and $g$ are respectively finitevalued. Then $U \cap V$ is open and dense, and $f+g$ and $f g$ are obtained by taking the unique elements of $C_{*}(S)$ which agree with the pointwise sum and product on $U \cap V$. If the zeros of $f$ are also nowhere dense, the function $1 / f$ is similarly defined. We now have the necessary notation and background to study dimension functions. 
Trrorem 4.5. To each element $u$ of $J$ with $|u|=1$ there is associated, isniquely, a function $d: J \rightarrow C_{*}(S)^{+}$with the following properties:

(i) $d(u)=1$,

(ii) for $x \in J$, the support of $d(x)$ is $\sigma(|x|)$,

(iii) for $x$ and $y$ in $J, x \sim y$ if and only if $d(x)=d(y)$,

(iv) for $x \in J$ and $e \in E, d(e \wedge x)=\phi(e) d(x)$,

(v) if $x \in J$ and $y \in J, d(x \vee y)+d(x \wedge y)=d(x)+d(y)$,

(vi) if $\left\{x_{i}: i \in I\right\}$ is an increasing directed set with least upper bound $x \in J$, then $d\left(x_{i}\right) \uparrow d(x)$.

Proof. Let $a \in J$ with $a \geqq u$. Then by Theorem 4.4 the center of $[0, a]$ is naturally isomorphic to $E$, so the dimension function $D_{a}$ induced by perspectivity on $[0, a]$ may be assumed to take values in $C_{*}(S)^{+}[14, \mathrm{p} .111]$. Applying Satz 1.4, Hilfssatz 1.4 and Hilfssatz 1.5 on pp. 112-115 of [14], we see that $D_{a}$ satisfies (ii)-(vi) on $[0, a]$. Hence the set of zeros of $D_{a}(u)$ is nowhere dense, and the equation $d_{a}(x)=\left(1 / D_{a}(u)\right) D_{a}(x)$ defines a function $d_{a}:[0, a] \rightarrow C_{*}(S)^{+}$with $d_{a}(u)=1$. Now (ii) -(v) clearly hold for $d_{a}$ on $[0, a]$. To verify (vi), recall that $D_{a}\left(x_{i}\right) \uparrow D(x)$ in the lattice $C_{*}(S)^{+}$ implies that $D_{a}\left(x_{i} ; s\right) \uparrow D_{a}(x ; s)$ for a residual set of $s \in S$ and hence $\left(1 / D_{a}(u)\right)(s) D_{a}\left(x_{i} ; s\right) \uparrow\left(1 / D_{a}(u)\right)(s) D_{a}(x ; s)$ for a (perhaps smaller) residual set of $s \in S$; thus $\left(1 / D_{a}(u)\right) D\left(x_{i}\right) \uparrow\left(1 / D_{a}(u)\right) D(x)$. If $d^{\prime}$ is another function $[0, a] \rightarrow C_{*}(S)^{+}$satisfying (i)-(vi) then setting $D^{\prime}(x)=\left(1 / d^{\prime}(a)\right) d^{\prime}(x)$ defines a function satisfying (ii)-(vi) for which $D^{\prime}(a)=1$. By Satz 3.3 on p. 129 of $[14], D^{\prime}=D_{a}$. Since $(1 / d(a))=D^{\prime}(u)=D_{a}(u)$, it follows that $d^{\prime}=d_{a}$. (Cf. the corollary of Theorem 5.3 in [15].) Now define $d: J \rightarrow C_{*}(S)^{+}$ by taking $d(x)=d_{a}(x)$ for any $a \geqq u \bigvee x$; the above argument shows that $d_{a}$ and $d_{b}$ agree on $[0, a \wedge b]$, so $d$ is well-defined. Properties (i)-(vi) follow from properties (i)-(vi) for the $d_{a}$, and the uniqueness of $d$ follows from the uniqueness of each $d_{a}$. Q.E.D.

Fix an arbitrary $u \in J$ with $|u|=1$, and let $d: J \rightarrow C_{*}(S)^{+}$be given by the theorem. Extend $d$ to a function from $L$ to $C^{*}(S)^{+}$by defining

$$
d(a)=\sup \left\{d(x): x \in J_{0}(a)\right\},
$$

the supremum being taken in $C^{*}(S)^{+}$. If $d$ is to maintain property (vi) on $L$, this equation must hold. This function $d$ will be called the dimension function on $L$ determined by $u$. We now investigate the properties of $d$. Let us write $d(a ; s)$ for the value of $d(a)$ at $s$.

Lemma 4.6. If $a \in L$ and $e \in E$, then $d(e \wedge a)=\phi(e) d(a)$.

Proof. This follows immediately from Theorem 4.5 (iv) once one notices that $J_{0}(e \wedge a)=\left\{e \wedge x: x \in J_{0}(a)\right\}$.

Lemma 4.7. If $a \in L$ and $t_{1}, t_{2}, \cdots$ are all in $J_{0}(a)$ and if $d\left(t_{n}\right) \geqq d(a)$ $-1 / n$ for $n=1,2, \cdots$, then $a=\sup \left\{t_{n}: n \geqq 1\right\}$. 
Proof. First notice that $d(a) \in C_{*}(S)$, i.e., $d(a)$ is finite-valued on an open everywhere dense set. Set $x=\sup \left\{t_{n}: n=1,2, \cdots\right\}$; then $x \leqq a$. If $a>x$, then there is a nonzero $y \in J_{0}(a-x)$. Thus there must be an $s \in S$ and an integer $k \geqq 1$ such that $1 / k<d(y ; s)<+\infty$ and $d(a ; s)<+\infty$. But $d(a ; s) \geqq d\left(t_{k}+y ; s\right)=d\left(t_{k} ; s\right)+d(y ; s)=d(a ; s)-1 / k+d(y ; s)$ $>d(a ; s)$. This contradiction proves $x=a$. Q.E.D.

The following piecing-together process is useful.

Definition 4.8. Let $\left\{x_{i}: i \in I\right\}$ be an indexed set in $L$. An element $y \in L$ will be called a mosaic of the set or of the $x_{i}$ 's if there are an orthogonal subset $T$ of $E$ and a function $\mu: T \rightarrow I$ such that $\Sigma T=1$ and

$$
y=\Sigma\left\{e \wedge x_{\mu(e)}: e \in T\right\} .
$$

The definition of course applies with only a minor change to nonindexed sets. Also the given set is always contained in the set of its mosaics. Taking an analogy with measurable functions from a sequence of functions converging in measure, one can construct a sequence (of mosaics of the given sequence) which converges uniformly a.e. This is the type of change in behavior we shall make by taking mosaics.

Lemma 4.9. Let $\left\{x_{i}: i \in I\right\}$ be directed upward in $L$ and let

$$
f=\sup \left\{d\left(x_{i}\right): i \in I\right\},
$$

in $C^{*}(S)^{+}$. Define a sequence $f_{1}, f_{2}, \cdots$ in $C_{*}(S)$ by setting $f_{n}(s)=f(s)-1 / n$ if $s \in F$, where $F$ is the closure of the set where $f$ is finite, and $f_{n}(s)=n$ if $s \in S-F$. Then there is an increasing sequence of mosaics of the $x_{i}$ 's, say $y_{1}, y_{2}, \cdots$, such that $f_{n} \leqq d\left(y_{n}\right) \leqq f$ for each integer $n=1,2, \ldots$.

Proof. The set of mosaics is also directed upward and $d(y) \leqq f$ for any mosaic $y$, so it suffices to find a mosaic $y_{n}$ with $f_{n} \leqq d\left(y_{n}\right)$.

Let $T$ be the set of elements $e \in E$ for which there is an $i \in I$ such that $d\left(x_{i}\right) \geqq f_{n}$ on $\sigma(e)$, and let $T_{0}$ be a maximal orthogonal subset of $T$. The maximality of $T_{0}$ implies $\Sigma T_{0}=1$, since the set of $s \in S$ for which $f(s)$ $=\sup \left\{d\left(x_{i} ; s\right): i \in I\right\}, f_{n}(s)<+\infty$, and $f_{n}(s)+1 / n \leqq f(s)$, is a residual set. By the choice of $T_{0}$, there is a function $\mu: T_{0} \rightarrow I$ such that $d\left(x_{\mu(e)}\right) \geqq f_{n}$ on $\sigma(e)$ whenever $e \in T_{0}$. Let $y_{n}$ be the mosaic constructed using $T_{0}$ and $\mu$. Then for $e \in T_{0}, e \wedge y_{n}=e \bigwedge x_{\mu(e)}$ so $d\left(y_{n}\right) \geqq f_{n}$ on $\sigma(e)$. Hence $d\left(y_{n}\right) \geqq f_{n}$.

Corollary 4.10. Let $a \in L$ and suppose $d(a)$ is in $C_{*}(S)$. Then there is an increasing sequence $t_{1}, t_{2}, \ldots$ in $J_{0}(a)$ such that for each $n$ we have $d\left(t_{n}\right)$ $\geqq d(a)-1 / n$ and hence also such that $a=\sup \left\{t_{n}: n=1,2, \cdots\right\}$.

Proof. The hypotheses of the lemma are satisfied by $J_{0}(a)$ and $f=d(a)$, and we have $f_{n}=d(a)-1 / n$ in this case. The set of mosaics of $J_{0}(a)$ is just $J_{0}(a)$, as in the proof of Theorem 4.4. The proof is completed by applying Lemma 4.9 and Lemma 4.7 in that order. Q.E.D. 
Now we begin the attack on the properties of $d$ needed to prove the dimension lattice axioms. Notice that we are proving from the lattice structure alone the properties which hold for a dimension function on an axiomatic dimension lattice (see [9] and [15]). In connection with the next lemma, note that $x^{\prime}$ is a complement of $y$ if and only if $y^{\prime}$ is a complement of $x$.

LEMMA 4.11. If $x$ and $y$ are in $J$ and $y^{\prime}$ is a complement of $x$ then $x \sim y$.

Proof. Set $y_{1}=(x \bigvee y) \wedge y^{\prime}=y^{\prime}-\left(x^{\prime} \wedge y^{\prime}\right)$, and let $\alpha$ be the isomorphism of $[0, x \bigvee y] \times\left[0, x^{\prime} \wedge y^{\prime}\right]$ into $L$ as in Lemma 2.1. Then $x \wedge y_{1} \leqq x$ $\bigwedge y^{\prime}=0$ since $y_{1} \leqq y$, and $\alpha\left(y_{1}, x^{\prime} \wedge y^{\prime}\right)=y^{\prime}$ and $\alpha(x, 0)=x$, so

$$
\begin{aligned}
1 & =x \bigvee y^{\prime} \\
& =\alpha\left(x \bigvee y_{1}, 0 \bigvee\left(x^{\prime} \wedge y^{\prime}\right)\right) \\
& =\alpha\left(x \bigvee y_{1}, x^{\prime} \wedge y^{\prime}\right) ;
\end{aligned}
$$

but $\alpha\left(x \bigvee y, x^{\prime} \wedge y^{\prime}\right)=1$, and $\alpha$ is one-one, so $x \bigvee y_{1}=x \bigvee y$. Thus $y_{1}$ is a complement of both $x$ and $y$ in $[0, x \vee y \mid$.

Lemma 4.12. If $x \in J, y \in L$, and $x^{\prime}$ is a complement of $y$, then $d(x)=d(y)$.

Proof. Let $t \in J_{0}(y)$ and set $r=\left(t \bigvee x^{\prime}\right) \wedge x$. Then $r \wedge x^{\prime}=0=t \wedge x^{\prime}$ because $r \leqq x$ and $t \leqq y$, and $r \bigvee x^{\prime}=t \bigvee x^{\prime}$ by weak modularity. In $\left[0, t \bigvee x^{\prime}\right], x^{\prime}$ is the orthocomplement of $r$, and applying Lemma 2.1 to $\left[0, t \bigvee x^{\prime}\right] \times\left[0,\left(t \bigvee x^{\prime}\right)^{\prime}\right]$ we see that $r^{\prime}=x^{\prime} \bigvee\left(t \bigvee x^{\prime}\right)^{\prime}$ is a complement of $t$. Since $r$ and $t$ are in $J$, by Lemma 4.11 we conclude $r \sim t$, so $d(r)=d(t)$. Thus $d(t) \leqq d(x)$, and hence $d(y) \leqq d(x)$, so $d(y) \in C_{*}(S)$. By Corollary 4.10, there is an increasing sequence $t_{1}, t_{2}, \ldots$ in $J$ such that $d\left(t_{n}\right) \uparrow d(y)$ and $t_{n} \uparrow y$. For each $n$, set $r_{n}=x \wedge\left(x^{\prime} \bigvee t_{n}\right)$. Then $r_{1}, r_{2}, \cdots$ is an increasing sequence. Now $t_{n}^{\prime} \wedge x \downarrow 0$ because $t_{n} \bigvee x^{\prime} \uparrow y \bigvee x^{\prime}=1$. Taking orthocomplements in $[0, x]$, we get $r_{n} \uparrow x$. Hence $d\left(r_{n}\right) \uparrow d(x)$, because $[0, x]$ is a von Neumann lattice. But $d\left(r_{n}\right)=d\left(t_{n}\right)$ just as $d(r)=d(t)$ above, and $d\left(t_{n}\right) \uparrow d(y)$, so $d(y)=d(x)$.

THEOREM 4.13. If $a$ and $b$ are in $L$, then $d(a-(a \wedge b))=d((a \bigvee b)-b)$.

Proof. Let $x \in J_{0}(a-(a \wedge b))$ and set $y=(b \bigvee x)-b$. Restricting to $[0, b \bigvee x], x$ and $y$ satisfy the hypotheses of Lemma 4.12 , so $d(x)=d(y)$ $\leqq d((a \bigvee b)-b)$. Thus $d(a-(a \wedge b)) \leqq d((a \bigvee b)-b)$. Applying this to $b^{\prime}$ and $a^{\prime}$, we see that $d\left(b^{\prime}-\left(b^{\prime} \wedge a^{\prime}\right)\right) \leqq d\left(\left(b^{\prime} \vee a^{\prime}\right)-a^{\prime}\right)$. But $b^{\prime}$ $-\left(b^{\prime} \wedge a^{\prime}\right)=(a \vee b)-b$ and $\left(b^{\prime} \vee a^{\prime}\right)-a^{\prime}=a-(a \wedge b)$, sod $(a-(a \wedge b))$ $\geqq d((a \vee b)-b)$.

LEMMA 4.14. If $a$ and $b$ are in $L$ and $a \perp b$, then $d(a+b)=d(a)+d(b)$.

Proof. Clearly $d(a+b) \geqq d(a)+d(b)$. To prove the reverse inequality, 
we first suppose $a \in J$. If $t \in J_{0}(a+b)$, then $((a \bigvee t)-a) \in J_{0}(b)$, so $d(t)$ $\leqq d(a \bigvee t)=d(a)+d((a \bigvee t)-a) \leqq d(a)+d(b)$. Thus $d(a+b) \leqq d(a)$ $+d(b)$ for $a \in J$, so $d(a+b)=d(a)+d(b)$ for $a \in J$.

Consider now the general case. Let $x \in J_{0}(a+b)$. By the first case, and since $(a \bigvee x)-a \leqq b$, we have

$$
\begin{aligned}
d(x) & \leqq d(a \bigvee x) \\
& \leqq d(x)+d((a \bigvee x)-x) \\
& =d(x-(a \wedge x))+d(a \wedge x)+d((a \bigvee x)-x) \\
& =d((a \bigvee x)-a)+d(a \wedge x)+d(a-(a \wedge x)) \\
& =d((a \bigvee x)-a)+d(a) \\
& \leqq d(b)+d(a)
\end{aligned}
$$

Hence $d(a+b) \leqq d(a)+d(b)$ in general. Q.E.D.

The next theorem is needed to prove axiom $\mathrm{D}^{\prime}$ and is also essential in the proofs of several facts about $J$.

Theorem 4.15. If $a$ and $b$ are in $L$, then $d(a \bigvee b)+d(a \wedge b)=d(a)+d(b)$.

Proof. Apply Theorem 4.13 and then Lemma 4.14.

Now let us prove the above-mentioned facts about $J$.

Theorem 4.16. The ideal $J$ is the set of all $a \in L$ for which $d(a) \in C_{*}(S)$. If $a \in J$ and $b \in L$, and if $a$ and $b$ have $a$ common complement, then $b \in J$ and $a \sim b$.

Proof. The set of all $x \in L$ with $d(x) \in C_{*}(S)^{+}$is an ideal by Theorem 4.15. This ideal has a positive valuation into $C_{*}(S)$, namely $d$, and can be proved to be modular by the argument used to prove that a lattice with a positive real-valued valuation must be modular $[2$, p. 76]. This ideal contains $J$ and hence is equal to $J$. The rest now follows from Lemma 4.12 and Theorem 4.5.

Now we can prove that $J$ is unique.

Theorem 4.17. The ideal $J$ is the set of all $a \in L$ such that $[0, a]$ is modular. Thus $J$ is the largest modular ideal in $L$, and hence is unique.

Proof. We need only prove that $a \in J$ if $[0, a]$ is modular, so suppose $[0, a]$ is modular. Then as in Theorem 4.4, we can choose first a $b \in J_{0}(a)$ with $|b|=|a|$, and then a $c \in J_{0}\left(|b|^{\prime}\right)$ with $|c|=|b|^{\prime}$. Then $b+c \in J$ and $|b+c|=1$, so $b+c$ may be taken as the element $u$ used in constructing the "dimension function" $d$. The center of $[0, b]$ is naturally isomorphic to the center of $[0, a]$ and to $[0,|b|] \cap E$, both by Theorem 4.3. Thus $\sigma(|a|)$ is naturally identified with the Stone space of the center of $[0, a\rfloor$, and the restriction of $d$ to $[0, a\rfloor$ may be regarded as having values in $C^{*}(\sigma(|a|))^{+}$. 
Let $d_{1}$ be the dimension function induced by perspectivity on $[0, a]$ such that $d_{i}(b)=1$. That this exists is seen as in the proof of Theorem 4.5, and as in the proof of Theorem 4.5, $d_{1}$ and $d$ must agree on $[0, y]$ whenever $b \leqq y \in J_{0}(a)$. Furthermore, if $x_{i} \uparrow x \leqq a$ then $d_{1}\left(x_{i}\right) \uparrow d_{1}(x)$, so $d_{1}(a)$ $=\sup \left\{d_{1}(x): x \in J_{0}(a)\right\}=\sup \left\{d(x): x \in J_{0}(a)\right\}=d(a)$. Thus $d(a)$ $\in C_{*}(S)$, so $a \in J$.

These theorems will now be used to prove further properties of $d$. The first of these would follow immediately from Theorem 4.15 and $d(0)=0$, if $d(1)$ were in $C_{*}(S)$.

THEOREM 4.18. If elements $a$ and $b$ in $L$ have a common complement, then $d(a)=d(b)$.

Proof. For every $e \in E, a \wedge e$ and $b \wedge e$ have a common complement. Let $e_{1}$ be the largest element of $E$ such that $a \wedge e_{1} \in J$. By Theorem 4.16, $e_{1}$ is also the largest element of $E$ such that $b \wedge e_{1} \in J$, and $d\left(a \wedge e_{1}\right)$ $=d\left(b \wedge e_{1}\right)$. If $d(a)$ took a finite value on $\sigma\left(1-e_{1}\right)$ then $d(a)$ would be bounded on a nonempty $\sigma(e)(e \in E)$ not contained in $\sigma\left(e_{1}\right)$. By Theorem 4.16, this contradicts the choice of $e_{1}$, so $d(a)$ is identically $+\infty$ on $\sigma\left(1-e_{1}\right)$. Similarly $d(b)$ is identically $+\infty$ on $\sigma\left(1-e_{1}\right)$.

Lemma 4.19. If $a \in L$ and $d(a)=+\infty$, then there is an orthogonal sequence $x_{1}, x_{2}, \ldots$ in $J_{0}(a)$ such that each $d\left(x_{n}\right) \geqq 1$.

Proof. Let $T$ be a maximal orthogonal subset of $\left\{x \in J_{0}(a): d(x) \geqq 1\right\}$. It will suffice to prove that $T$ is infinite. If $T$ were finite we would have $x=\Sigma T \in J_{0}(a)$, so $d(x) \in C_{*}(S)^{+}$. Now $d(a)=d(x)+d(a-x)$ by Lemma 4.14, so $d(a-x)=+\infty$. By Lemma 4.10 there is a $y \in J_{0}(a-x)$ with $d(y) \geqq 1$, and thus $T \cup\{y\}$ contradicts the maximality of $T$. Hence $T$ is infinite.

THEOREM 4.20. Suppose $a$ and $b$ are in $L$ with $d(a)=d(b)$ and $b=b_{1}+b_{2}$. Then $a=a_{1}+a_{2}$ with $d\left(a_{1}\right)=d\left(b_{1}\right)$ and $d\left(a_{2}\right)=d\left(b_{2}\right)$.

Proof. Let $e_{1}$ be the largest element of $E$ for which $b_{1} \wedge e_{1} \in J$ ( $e_{1}$ is the largest element of $E$ for which $\left.d\left(b_{1} \wedge e_{1}\right) \in C_{*}(S)\right)$, and similarly choose $e_{2}$ for $b_{2}$. By restricting to $\left[0, e_{1} \wedge e_{2}\right],\left[0, e_{1} \wedge e_{2}^{\prime}\right],\left[0, e_{2} \wedge e_{1}^{\prime}\right]$ and $\left[0, e_{1}^{\prime} \wedge e_{2}^{\prime}\right]$ and noticing the symmetry of the situations in $\left[0, e_{1} \wedge e_{2}^{\prime}\right]$ and $\left[0, e_{2} \wedge e_{1}^{\prime}\right]$, we may consider the following cases separately: (i) $d(a)=d(b) \in C_{*}(S)$, (ii) $d\left(b_{1}\right) \in C_{*}(S)$ but $d(a)=d(b)=d\left(b_{2}\right)=+\infty$, and (iii) $d\left(b_{1}\right)=d\left(b_{2}\right)$ $=d(a)=d(b)=+\infty$.

In Case (i), $a \bigvee b \in J$ so $\{0, a \bigvee b\rfloor$ is a von Neumann lattice and the result follows immediately from Satz 1.4, p. 112 of [14].

Case (ii). Let $y_{1}, y_{2}, \ldots$ be an increasing sequence in $J_{0}(a)$ such that for every $n$ we have $d\left(y_{n}\right) \geqq n$ (Lemma 4.9). For each $n \geqq 1$, choose $e_{n} \in E$ so that $\sigma\left(e_{n}\right)$ is the interior of the set on which $d\left(b_{1}\right) \leqq n$. Then 


$$
\bigcup\left\{\sigma\left(e_{n}\right): n=1,2, \cdots\right\}
$$

is an open everywhere dense set. Set $e_{0}=0$, and set

$$
y=\Sigma\left\{y_{n} \wedge\left(e_{n}-e_{n-1}\right): n=1,2, \cdots\right\} \text {. }
$$

Then $y$ is in $J_{0}(a)$, and $d(y) \geqq d\left(b_{1}\right)$. Now $\left[0, b_{1} \bigvee y\right]$ is a von Neumann lattice because $b_{1} \bigvee y \in J$, so there is an $a_{1} \leqq y$ with $d\left(a_{1}\right)=d\left(b_{1}\right)$. Both $d\left(a-a_{1}\right)$ and $d\left(b_{2}\right)$ are then identically infinite, so we may take $a_{2}=a-a_{1}$.

Case (iii). Let $x_{1}, x_{2}, \ldots$ be an orthogonal sequence in $J_{0}(a)$ with $d\left(x_{n}\right)$ $\geqq 1$ for each $n$. Set $a_{1}=\Sigma\left\{x_{2 k}: k \geqq 1\right\}$ and $a_{2}=a-a_{1}$. Then $d\left(a_{1}\right)$ and $d\left(a_{2}\right)$ are identically $+\infty$ and $a=a_{1}+a_{2 \cdot}$. Q.E.D.

As the last fundamental fact, we prove that $d$ is completely additive on orthogonal sets. Since we already know it is finitely additive, the following theorem implies the desired result. The proof of this theorem for the type I case was in the author's thesis, but the proof in the general case was discovered only after receiving a private communication from M. D. MacLaren which stated a theorem giving a lattice theoretic characterization of locally finite dimension lattices which is different from the one given in the present paper. His result, combined with the results of $\$ 5$, provides another proof of part (i) of Theorem 4.23.

THEOREM 4.21. If $\left\{x_{i}: i \in I\right\}$ is an increasing directed family in $L$ with least upper bound $x$, then $d\left(x_{i}\right) \uparrow d(x)$ in $C^{*}(S)$.

Proof. Let $f=\sup \left\{d\left(x_{i}\right): i \in I\right\}$ in $C^{*}(S)$. For $e \in E$, then $\phi(e) \cdot f$ is the least upper bound of the $d\left(e \wedge x_{i}\right)$ and $e \wedge x_{i} \uparrow e \wedge x$. Let $e_{1}$ be the largest element of $E$ such that $e_{1} \wedge x \in J$, and let $e_{2}$ be the largest element of $E$ such that $f$ is identically $+\infty$ on $\sigma\left(e_{2}\right)$. Since $f \leqq d(x)$, we have $e_{1} \perp e_{2}$. Since $\left[0, e_{1} \wedge x\right]$ is a von Neumann lattice, we have $\phi\left(e_{1}\right) \cdot f=d\left(e_{1} \wedge x\right)$, and clearly $\phi\left(e_{2}\right) \cdot f=d\left(e_{2} \wedge x\right)$. Thus the proof will be complete if $e_{1}+e_{2}$ $=1$. This will follow if $x \wedge e \in J$ whenever $f$ is bounded on $\sigma(e)$, so it suffices to prove that $x \in J$ if $f$ is bounded. If $\sigma\left(e_{0}\right)$ is the interior of the zero set of $f$, then $x \wedge e_{0}=0$. Now let $T$ be a maximal orthogonal subset of $\{e \in E: f$ is bounded away from 0 on $\sigma(e)\}$. Then $\bigcup\{\sigma(e): e \in T\}$ is dense in the support of $f$; furthermore $x$ will be in $J$ if $e \in T$ implies $x \wedge e \in J$. Hence we may suppose there is an $\epsilon>0$ such that $f \geqq \epsilon$.

Let $y_{1}, y_{2}, \cdots$ be an increasing sequence of mosaics of the $x_{i}^{\prime}$ 's, such that for each $n$, we have $d\left(y_{n}\right) \geqq f-1 / n$. Let $y=\sup \left\{y_{n}: n=1,2, \cdots\right\}$. Then $y \leqq x$, and if $y<x$ there is an $i \in I$ with $x_{i}>x_{i} \wedge y$, so $z_{i}=x_{i}-\left(x_{i} \wedge y\right)$ $>0$. Then for any $n \geqq 1$ we have $f \geqq d\left(y_{n} \vee x_{i}\right)$ because the mosaics are directed and each $x_{i}$ is a mosaic, but $d\left(y_{n} \vee x_{i}\right) \geqq d\left(y_{n}\right)+d\left(z_{i}\right) \geqq f-1 / n$ $+d\left(z_{i}\right)$ and not every $d\left(z_{i}\right)-1 / n \leqq 0$. Thus $y=x$.

Choose now an $n \geqq 2 / \epsilon$ and set $t_{0}=y_{n}, t_{1}=y_{n+1}-y_{n}, t_{2}=y_{n+2}-y_{n+1}, \cdots$. Then for any $m \geqq 1$ we have $d\left(t_{1}+\cdots+t_{m}\right) \leqq \epsilon / 2 \leqq d\left(t_{0}\right)$. Thus there 
is an orthogonal sequence $r_{1}, r_{2}, \ldots$ in $\left\lfloor 0, t_{0}\right\rfloor$ with $r_{1} \sim t_{1}, r_{2} \sim t_{2}, \ldots$. For distinct $m$ and $k$, we have $r_{m} \perp r_{k}, t_{m} \perp t_{k}, r_{m} \perp t_{k}$ and $t_{m} \perp r_{k}$. Thus $r_{m} \bigvee t_{m}$ $\perp r_{k} \bigvee t_{k}$. Also, for every $m$ there is a $u_{m}$ in $\left\lfloor 0, r_{m} \bigvee t_{m}\right\rfloor$ with $u_{m} \wedge r_{m}=u_{m} \wedge t_{m}$ $=r_{m} \wedge t_{m}=0$ and $u_{m} \bigvee r_{m}=u_{m} \bigvee t_{m}=r_{m} \bigvee t_{m}$. Set $r=\Sigma\left\{r_{m}: m=1,2, \cdots\right\}$, $t=\Sigma\left\{t_{m}: m=1,2, \cdots\right\}$, and $u=\Sigma\left\{u_{m}: m=1,2, \cdots\right\}$. Then by Lemma 2.1, $u \wedge r=u \wedge t=r \bigwedge t=0$ and $u \bigvee r=u \bigvee t=r \bigvee t$ (cf. Theorem 3 of [6]). Since $r \in J$, this implies $t \in J$, by Theorem 4.16. Thus $x=t_{0}+t$ is in J. Q.E.D.

ThEOREM 4.22. If $\left\{x_{i}: i \in I\right\}$ is an orthogonal set in $L$ with least upper bound $x$, then

$$
d(x)=\Sigma\left\{d\left(x_{i}\right): i \in I\right\} .
$$

Proof. The sum on the right-hand side is defined to be the least upper bound of the finite sums, and $d\left(x_{i_{1}}+\cdots+x_{i_{n}}\right)=d\left(x_{i_{1}}\right)+\cdots+d\left(x_{i_{n}}\right)$, so the equality follows directly from Theorem 4.21.

This completes our study of $d$, and we have shown that the basic properties extend from the modular case to the locally modular case. Now these facts can be used to prove a theorem about dimension lattices.

THEOREM 4.23. Let $L$ be a complete orthocomplemented weakly modular lattice. If $L$ is locally modular, then there exists a dimensional equivalence relation $\sim$ on $L$ such that:

(i) $(L, \sim)$ is a locally finite dimension lattice,

(ii) an element $a \in L$ is finite with respect to $\sim$ if and only if $[0, a\rfloor$ is modular,

(iii) an element $e \in L$ is invariant with respect to $\sim$ if and only if $e$ is in the center of $L$,

(iv) if $a$ and $b$ are finite with respect to $\sim$, then $a \sim b$ if and only if $a$ and $b$ are perspective.

Proof. By Theorem 4.17, the set $J$ of all elements $a \in L$ for which $[0, a]$ is modular is an ideal and is join-dense. Take an element $u \in J$ with $|u|=1$, by Theorem 4.4, and define $d$ on $J$ as in Theorem 4.5. Now extend $d$ to $L$ as it was done preceding Lemma 4.6, and define $a \sim b$ to mean $d(a)=d(b)$. Then axiom $\mathrm{A}$ is clear, and axioms $\mathrm{B}, \mathrm{C}$ and $\mathrm{D}^{\prime}$ are contained in Theorems $4.20,4.22$ and 4.18 , respectively. Thus $(L, \sim)$ is a dimension lattice. Theorem 4.16 implies that $J$ is the set $\left\{a \in L: d(a) \in C_{*}(S)\right\}$. Since $C_{*}(S)$ is a vector lattice, and $b<a$ implies $d(a)=d(b)+d(a-b)$ and $d(a-b)$ $\neq 0$, every element of $J$ is finite. But if $d(a)$ is infinite on a nonempty open set, i.e., $d(a) \notin C_{*}(S)$, Lemma 4.19 makes it possible to construct $b<a$ with $d(b)=d(a)$. This proves (ii). Since $J$ is join-dense, we see that $L$ is locally finite. Now if $e$ is central and $a \prec e$, then $d(a ; s)=0$ for $s$ not in $\sigma(e)$. Thus for $x \in J_{0}(a)$, we have $d(e \wedge x)=\phi(e) \cdot d(x)=d(x)$ so $e \wedge x=x$; 
hence $e \wedge a=a$ and $a \leqq e$. This proves (iii), leaving only (iv). But Theorem 4.5 (iii) says that for elements of $J, d(a)=d(b)$ implies $a$ and $b$ are perspective in $[0, a \bigvee b]$, and if $a$ and $b$ are perspective in $[0, a \bigvee b]$ they are perspective in $L$, so $a \in J, b \in J$ and $d(a)=d(b)$ imply $a$ is perspective to $b$. The converse part of (iv) is just axiom $\mathrm{D}^{\prime}$.

CoROLLARY 4.24. If $L$ is a complete orthocomplemented weakly modular lattice, then there exists a locally finite-dimensional equivalence relation on $L$ if and only if $L$ is locally modular.

5. Semi-modularity. A pair $(x, y)$ is a modular pair, written $(x, y) M$, if $x \geqq z$ implies $x \wedge(y \bigvee z)=(x \wedge y) \bigvee z$. A pair $(x, y)$ is a dual-modular pair, written $(x, y) D M$, if $x \leqq z$ implies $(x \bigvee y) \wedge z=x \bigvee(y \wedge z)$. (See [2, p. 100], $[10$, p. 166].) We shall say that a lattice is semi-modular to signify that the relation $(x, y) M$ is symmetric, i.e., $(x, y) M$ implies $(y, x) M[2$, p. 101]. In an orthocomplemented lattice, $(x, y) M$ if and only if $\left(x^{\prime}, y^{\prime}\right) D M$, so an orthocomplemented lattice is semi-modular if and only if the relation $(x, y) D M$ is symmetric. Let $x \leqq z$ and set $z_{1}=(x \bigvee y) \wedge z$. Then $y \wedge z_{1}$ $=y \wedge z$, so $(x \bigvee y) \wedge z=x \bigvee(y \wedge z)$ if and only if $z_{1}=(x \bigvee y) \wedge z_{1}$ $=x \bigvee\left(y \wedge z_{1}\right)$. Thus to test the pair $(x, y)$ for dual modularity we need only consider elements $z \in[x, x \vee y]$.

Let $L$ be a locally modular complete oc.w.m. lattice. We shall prove that $L$ is semi-modular, using a proof analogous to the proof of Theorem III-6 of [10]. By Theorem 4.17, the set $J=\{x \in L:[0, x]$ is modular $\}$ is a join-dense ideal and is the largest modular ideal. As in $\$ 4$, write $J_{0}(x)$ for $J \wedge[0, x]$ whenever $x \in L$.

Lemma 5.1. Let $a \in J$ and let $b, c \in L$ with $a \leqq c$. Then $a \bigvee(b \wedge c)$ $=(a \bigvee b) \wedge c$. Thus $a \in J$ and $b \in L$ implies $(a, b) D M$; hence also $(a, b) M$, since $a \geqq z$ implies $z \in J$.

Proof. By Lemma 2.1, $x \rightarrow(b \wedge c) \bigvee x$ is an isomorphism of

$$
[0,(b \bigvee c)-(b \wedge c)]
$$

onto $[b \wedge c, b \bigvee c]$ and hence provides the latter with a join-dense modular ideal. Set $a_{1}=a \bigvee(b \wedge c)$. Then $\left[b \wedge c, a_{1}\right]$ is modular by Theorem 4.16 since $a_{1}-b \wedge c \sim a-a \wedge b \wedge c$. Also $\left(a_{1} \bigvee b\right) \wedge c=a_{1}$ if and only if $a \bigvee(b \wedge c)=(a \bigvee b) \wedge c$. Set $a_{2}=\left(a_{1} \bigvee b\right) \wedge c$; then $a_{2} \geqq a_{1}$. Also $a_{1} \bigvee b$ $=a_{2} \bigvee b$ and $a_{1} \wedge b=a_{2} \wedge b=b \wedge c$, so $a_{1}$ and $a_{2}$ are perspective in $[b \wedge c, b \bigvee c]$. Hence $a_{1}-b \wedge c$ and $a_{2}-b \wedge c$ are perspective in $L$. Since $a_{1}-b \wedge c \in J$, Theorem 4.16 shows that $a_{2}-b \wedge c \in J$, and since $a_{2}-b \wedge c$ $\geqq a_{1}-b \wedge c, a_{2}-b \wedge c=a_{1}-b \wedge c$; thus $a_{2}=a_{1}$. Q.E.D.

For ideals $K_{1}$ and $K_{2}$ of $L$, let $K_{1} \bigvee K_{2}$ denote the ideal generated by $K_{1}$ and $K_{2}$. The next lemma is the analogue of the fact that the vector sum of a finite-dimensional subspace and a closed subspace is closed. 
LemmA 5.2. If $a \in L$ and $c \in J$ then $J_{0}(a \bigvee c)=J_{0}(a) \bigvee J_{0}(c)$.

Proof. Clearly $J_{0}(a) \bigvee J_{0}(c) \subseteq J_{0}(a \bigvee c)$, so let $x \in J_{0}(a \bigvee c)$. Then $x \bigvee c \in J_{0}(a \bigvee c)$ and $(x \bigvee c) \wedge a \in J_{0}(a)$. By Lemma 5.1, $(a \wedge(x \bigvee c)) \bigvee c$ $=(x \bigvee c) \wedge(a \bigvee c)=x \bigvee c$, so $x \bigvee c \in J_{0}(a) \bigvee J_{0}(c)$. Hence $x \in J_{0}(a)$ $\bigvee J_{0}(c)$.

THEOREM 5.3. The following conditions are equivalent for elements $a$ and $b$ of $L$

(i) $J_{0}(a \bigvee b)=J_{0}(a) \bigvee J_{0}(b)$,

(ii) $J_{0}(a) \bigvee J_{0}(b)$ is join-dense in $[0, a \bigvee b]$,

(iii) $(a, b) D M$.

Proof. Clearly (i) implies (ii). (ii) implies (iii): Let $a \leqq c \leqq a \bigvee b$. We must prove $c=a \bigvee(b \wedge c)$, which will be true if $J_{0}(a) \bigvee J_{0}(b \wedge c)$ is joindense in $[0, c]$. Let $0<z \leqq c$. Now $c \leqq a \bigvee b$ and we have assumed $J_{0}(a)$ $\bigvee J_{0}(b)$ is join-dense in $[0, a \bigvee b]$, so there can be chosen $x \in J_{0}(a)$ and $y \in J_{0}(b)$ with $(x \bigvee y) \wedge z \neq 0$. Set $z_{1}=(x \vee y) \wedge z$; using modularity in $J$, we compute

$$
\begin{aligned}
x \bigvee\left(y \wedge\left(x \bigvee z_{1}\right)\right) & =(x \bigvee y) \wedge\left(x \bigvee z_{1}\right) \\
& =x \bigvee z_{1}
\end{aligned}
$$

Since $y \wedge\left(x \vee z_{1}\right) \leqq b \wedge c$, this shows that $x \bigvee z_{1}$ is in $J_{0}(a) \bigvee J_{0}(b \wedge c)$, and hence so is $z_{1}$. Thus for nonzero $z \in[0, c],\left(J_{0}(a) \vee J_{0}(b \wedge c)\right) \cap[0, z]$ $\neq\{0\}$, which is what we were to prove.

(iii) implies (i): Clearly $J_{0}(a) \bigvee J_{0}(b) \subseteq J_{0}(a \bigvee b)$, so suppose $x \in J_{0}(a \bigvee b)$ Set $x_{1}=a \bigvee x, x_{2}=x_{1}-a$, and $x_{3}=\left(b \wedge x_{1}\right)-(a \wedge b)$. Then $x-a \wedge x$ and $x_{2}$ are perspective, having $a \bigvee(a \bigvee x)^{\prime}$ as a common complement (Lemma 2.1), so $x_{2} \in J$ by Theorem 4.16 . Since $(a, b) D M$, we may compute as follows:

$$
\begin{aligned}
x_{1} & =a \bigvee\left(b \wedge x_{1}\right) \\
& =a \bigvee\left[(a \wedge b) \bigvee\left(\left(b \wedge x_{1}\right)-(a \wedge b)\right)\right] \\
& =a \bigvee\left[(a \wedge b) \bigvee x_{3}\right] \\
& =a \bigvee x_{3} .
\end{aligned}
$$

From the first equality it follows that $x_{2}=x_{1}-a=\left(a \bigvee\left(b \wedge x_{1}\right)\right)-a$ and $x_{3}=\left(b \wedge x_{1}\right)-(a \wedge b)=b \wedge x_{1}-a \wedge b \wedge x_{1}$ are perspective. Hence $x_{3} \in J$ by Theorem 4.16, and since $x_{1}=a \bigvee x_{3}$, Lemma 5.2 implies that $J_{0}\left(x_{1}\right)$ $=J_{0}\left(a \bigvee x_{3}\right)=J_{0}(a) \bigvee J_{0}\left(x_{3}\right)$. Since $x \in J_{0}\left(x_{1}\right)$ and $J_{0}\left(x_{3}\right) \subseteq J_{0}(b)$, this shows that $x \in J_{0}(a) \bigvee J_{0}(b)$, as desired.

THEOREM 5.4. If $L$ is a complete orthocomplemented weakly modular lattice which is locally modular, then $L$ is semi-modular. 
Proof. The equivalence of conditions (i) and (iii) in Theorem 5.3 shows that the relation $(a, b) D M$ is symmetric.

REMARK. The proof that (i) and (iii) are equivalent is easily done without mentioning (ii), but it seems of interest to notice that (i) and (ii) are equivalent. In the atomic case (i) and (ii) are obviously the same.

6. Sufficient conditions for local modularity. The following is a standard covering condition $[2$, p. 100]:

if $x$ covers $x \wedge y$ then $x \bigvee y$ covers $y$.

For oc.w.m. lattices it is equivalent to this: if $x-x \wedge y$ is an atom then $x \bigvee y-y$ is an atom. An atom is always multiplicity free (m.f.) and the central cover of an atom is an atom in the center (\$2). On the other hand, if $0<x \leqq e$, if $x$ is m.f., and if $e$ is an atom in the center, then $x$ is an atom. Hence we may strengthen (p) as follows, for oc.w.m. lattices: if $x-x \wedge y$ is m.f. then $x \vee y-y$ is m.f.

Both (p) and $\left(\mathrm{p}^{+}\right)$are equivalent to their duals by orthocomplementation. Thus (p) implies both $\left(\xi^{\prime}\right)$ and $\left(\xi^{\prime \prime}\right)\left[2\right.$, p. 66]. A chain $c_{0}<c_{1}<\cdots<c_{k}$ is connected if $1 \leqq j \leqq k$ implies $c_{j}$ covers $c_{j-1}[2$, p. 11].

LEMMA 6.1. Let $M$ be an oc.w.m. lattice satisfying (p), in which every nonzero element dominates an atom, and suppose that $a_{0}=0<a_{1}<\cdots<a_{n}=1$ is a maximal chain in $M$. If $b_{0}=0<b_{1}<\cdots<b_{n-1}$ is a connected chain in $M$ then 1 covers $b_{n-1}$.

Proof. We proceed by induction on $n$. For $n=1$ the lemma is obvious; suppose it is proved for $n \leqq k$, and consider $n=k+1$. If $a_{k} \geqq b_{k}$, then by the case $n=k, a_{k}$ covers $b_{k-1}$. Hence $a_{k}=b_{k}$, giving the desired result. If $a_{k} b_{k}$, then $a_{k} \bigvee b_{k}>a_{k}$, so $a_{k} \bigvee b_{k}=1$. Now $a_{k} \bigvee b_{k}$ covers $a_{k}$, so $b_{k}$ covers $a_{k} \wedge b_{k}$, by the dual of (p). Let us construct a connected chain $c_{0}=0<c_{1}$ $<\cdots<c_{k-1}$ in $\left[0, a_{k} \wedge b_{k}\right]$. We may take $c_{1}$ to be an atom $\leqq a_{k} \wedge b_{k}$, and proceed by induction. Suppose we have chosen $c_{1}<c_{2}<\cdots<c_{j}$ connected, and $j<k-1$. If $c_{j}=a_{k} \wedge b_{k}$, then $c_{0}=0<c_{1}<\cdots<c_{j}<b_{k}$ is a maximal chain in $\left[0, b_{k}\right]$, and applying the case $n=j+1$ we contradict the existence of the connected chain $b_{0}=0<b_{1}<\cdots<b_{k}$. Thus $c_{j}<a_{k} \wedge b_{k}$ so $\left(a_{k} \wedge b_{k}\right)$ $\wedge c_{j}^{\prime}>0$ and we can choose an atom $p_{j} \leqq\left(a_{k} \wedge b_{k}\right) \wedge c_{j}^{\prime}$. By (p), $c_{j+1}$ $=c_{j} \bigvee p_{j}$ covers $c_{j}$, and $c_{0}=0<c_{1}<\cdots<c_{j+1}$ is connected. Taking the chain so constructed, the case $n=k$ applied to $\left[0, a_{k}\right]$ shows that $a_{k}$ covers $c_{k-1}$. Since $a_{k}>a_{k} \wedge b_{k} \geqq c_{k-1}$, we see that $a_{k}$ covers $a_{k} \wedge b_{k}$. Hence 1 $=a_{k} \bigvee b_{k}$ covers $b_{k}$.

Theorem 6.2. (CF. Lemma 4.2 of [13]). Let $M$ be an oc.w.m. lattice satisfying ( $\mathrm{p})$, in which every nonzero element dominates an atom, and suppose there is a finite maximal chain in $M$. Then $M$ is modular. 
Proof. If $a<b$, the method used in Lemma 6.1 can be used to construct connected chains in $[0, b-a \mid$. By Lemma 6.1 the lengths of such chains are bounded, so $[0, b-a\rfloor$ has a finite maximal chain. Since $[a, b]$ is isomorphic to $[0, b-a],[a, b]$ has a finite maximal chain. Thus every finite chain can be imbedded in a connected chain. Since every connected chain in $M$ is finite, by Lemma 6.1, every chain in $M$ is finite. Hence $M$ is modular by Theorem 3 , Chapter $\mathrm{V}$ of $[2]$.

THEOREM 6.3. Let $L$ be a complete oc.w.m. lattice containing an atom, and with center $=\{0,1\}$. Then $L$ is locally modular if and only if $(\mathrm{p})$ holds in $L$.

Proof. If $L$ is locally modular, then there exists a d.e.r. $\sim$ on $L$ by Theorem 4.23. Then for each $x$ and $y, x-x \wedge y \sim x \vee y-y$, so if $x-x \wedge y$ is an atom so is $x \bigvee y-y$. Thus (p) holds in $L$.

Now suppose that $L$ satisfies (p) and let $e$ be the join of all the atoms in $L$. If $x \in L$ and there is an atom $a$ not orthogonal to $x$, then $x \wedge\left(a \bigvee x^{\prime}\right)$ $=\left(a \bigvee x^{\prime}\right)-x^{\prime}$ is an atom by (p). Thus for any $x \in L, x \wedge e=0$ implies $x \perp e$, so $e$ is in the center of $L$. But $e>0$, so $e=1$, and $L$ is atomic. Let $J$ be the ideal in $L$ generated by the atoms. Then $J$ is join-dense since $L$ is atomic. If $a_{1}, \ldots, a_{k}$ are atoms in $L$ and $z=a_{1} \bigvee \ldots \bigvee a_{k}$, then (p) holds in $[0, z]$ because for $x$ and $y$ in $[0, z]$, the elements $x-x \wedge y$ and $x \vee y-y$ are the same whether calculated in $[0, z]$ or $L$. By Theorem 6.2, each such $[0, z]$ is modular, so $J$ is modular. Thus $L$ is locally modular. Q.E.D.

The next lemma follows immediately from Lemma 2.6.

Lemma 6.4. If $x$ is $m$.f. in $L$ and $y \leqq x \leqq z$, then $y$ is $m$.f. in $\lfloor 0, z\rfloor$.

Lemma 6.5. If $L$ is a complete oc.w.m. lattice satisfying $\left(\mathrm{p}^{+}\right)$, then the element $e=\sup \{x \in L: x$ is m.f. $\}$ is in the center of L. Furthermore, if $0<x$ $\leqq e$, then $x$ is a join of m.f. elements and there is at least one m.f. element $y \leqq x$ with $|y|=|x|$.

Proof. Let $x \in L$ be such that $y \leqq x$ implies $y$ is not m.f. If $z$ is m.f. and $x \not z$, then $x \wedge\left(x \wedge z^{\prime}\right)^{\prime}$ and $\left(x \bigvee z^{\prime}\right) \wedge\left(z^{\prime}\right)^{\prime}$ have a common complement (Corollary to Loomis' Lemma 41), the latter being nonzero because $x \bigvee z^{\prime}$ $>z^{\prime}$, and m.f. by Lemma 6.4. Thus $x \wedge\left(x \wedge z^{\prime}\right)^{\prime}$ is m.f. by $\left(\mathrm{p}^{+}\right)$, and we have contradicted our assumption about $x$. Thus $x$ is orthogonal to every multiplicity free element, so $x \perp e$. Now if $x \wedge e=0$, and $z$ is m.f., $x$ z; thus $x \wedge e=0$ implies $x \perp e$. Hence $e \in E$. The first assertion of the last sentence is an easy consequence of what we have already done. Note that if $T$ is a set of m.f. elements in $L$ and $x \neq y$ in $T$ implies $|x| \perp|y|$, then $\sup T$ is m.f. Using Zorn's lemma, we now can easily find a $y \leqq x$ with $|y|=|x|$ and $y$ m.f.

THEOREM 6.6. Let $L$ be a complete oc.w.m. lattice with a closed subalgebra, $E$, of the center, such that if $x-x \wedge y$ is m.f. with respect to $E$ then $x \bigvee y-y$ 
is $m$.f. with respect to $E$. If 1 is a finite join of elements which are $m . f$. with respect to $E$ then $L$ is modular.

Proof. We shall imbed $L$ as a sublattice of a direct product of modular lattices. Let $S$ be the Stone space of $E$, regarded for now only as the set of all maximal dual ideals of $E$. Given $s \in S$, define a congruence relation on $L$ by $x \equiv y$ if and only if there is an $e \in s$ with $x \wedge e=y \wedge e$. Let $L_{s}$ denote the quotient lattice, and $h_{s}$ the quotient homomorphism. Now any lattice homomorphic image of an oc.w.m. lattice can be given an orthocomplementation, uniquely, so that the homomorphism preserves orthocomplements. In that case the image is also weakly modular. Thus each $L_{s}$ is in a natural way oc.w.m. and hence so is $M=I I\left\{L_{s}: s \in S\right\}$. For $x$ in $L$ define $h(x)$ in $M$ by $(h(x))(s)=h_{s}(x)$. Then $h$ is an orthocomplementation preserving homomorphism. To prove that $h$ is one-one, it suffices to note that $h_{s}(x)>h_{s}(x \wedge y)$ if and only if $|x-(x \wedge y)| \in s$. The proof will be completed by showing that each $L_{s}$ satisfies the hypotheses of Theorem 6.2 and hence is modular.

First we prove that an element $a$ of $L_{s}$ is an atom if and only if there is an m.f. $x$ in $L$ with $|x| \in s$ and $h_{s}(x)=a$. Suppose that $|x| \in s$ and $h_{s}(x)=a$ is not an atom. Then there is $a b$ in $L_{s}$ with $0<b<a$. Choose $y$ so that $h_{s}(y)=b$, and set $y_{1}=x \wedge y$. Then $y_{1} \leqq x$ and $0<h_{s}\left(y_{1}\right)<h_{s}(x)$, so $\left|y_{1}\right| \in s$ and $\left|x-y_{1}\right| \in s$ and hence $e=\left|y_{1}\right| \wedge\left|x-y_{1}\right| \in s$. Hence $x$ is not m.f., by Lemma 2.6. Conversely, suppose that $|x| \in s$, and that $e \in s$ implies $x \wedge e$ is not m.f., i.e., suppose there is no m.f. element $z$ with $h_{s}(z)$ $=h_{s}(x)$. Choose $y \leqq x$ to be m.f. and with $|y|=|x|$. Then $0<h_{s}(y) \leqq h_{s}(x)$; and $h_{s}(y)<h_{s}(x)$, since otherwise there would be an $e \in s$ with $x \wedge e=y \wedge e$ and hence with $x \wedge e$ m.f. Thus $h_{s}(x)$ is not an atom. If $|x|$ is not in $s$, then $h_{s}(x)=0$, also not an atom. It now follows that every nonzero element of $L_{s}$ dominates an atom.

To show that (p) holds in $L_{s}$, let $a, b$ be in $L_{s}$ with a covering $a \wedge b$, and choose $x, y$ in $L$ with $h_{s}(x)=a, h_{s}(y)=b$. Since $a \wedge(a \wedge b)^{\prime}=h_{s}\left(x \wedge(x \wedge y)^{\prime}\right)$ is an atom, there is an $e \in s$ for which $(x \wedge e) \wedge((x \wedge e) \wedge(y \wedge e))^{\prime}$ $=\left(x \wedge(x \wedge y)^{\prime}\right) \wedge e$ is m.f. By $\left(\mathrm{p}^{+}\right)$, then $((x \wedge e) \vee(y \wedge e)) \wedge(y \wedge e)^{\prime}$ is m.f., so $(a \vee b) \wedge b^{\prime}=h_{s}\left(((x \wedge e) \vee(y \wedge e)) \wedge(y \wedge e)^{\prime}\right)$ is an atom.

Now if $x_{1}, \cdots, x_{n}$ are m.f. elements of $L$ with join 1 , the nonzero elements of $\left\{h_{s}\left(x_{i}\right): 1 \leqq i \leqq n\right\}$ are atoms with join 1 . By (p) it follows that $L_{s}$ has a finite maximal chain.

Theorem 6.7. Let $L$ be a complete oc.w.m. lattice with an m.f. element $u$ such that $|u|=1$. Then $L$ is locally modular if and only if $\left(\mathrm{p}^{+}\right)$holds in $L$.

Proof. If $L$ is locally modular, then there is a locally finite d.e.r. $\sim$ on $L$ such that every central element is invariant. Then an element $x$ is m.f. if and only if $y, z$ in $\mid 0, x\rfloor$ and $0<y_{1} \leqq y \perp z \geqq z_{1}>0$ implies $z_{1} \nsim y_{1}$. This 
property of $x$ is preserved under $\sim$, and always $x-x \wedge y \sim x \bigvee y-y$, so $\left(\mathrm{p}^{+}\right)$holds.

For the converse let $z$ be a finite join of elements which are m.f. with respect to the center, $E$. Notice that an element $x \leqq z$ is m.f. with respect to $E$ in $L$ if and only if it is m.f. with respect to $\{z \wedge e: e \in E\}$ in $[0, z]$. Thus by Theorem 6.6 the interval $\mid 0, z\rfloor$ is modular. The ideal generated by the m.f. elements is therefore modular, and the proof that it is join-dense is the same as the proof in Theorem 6.3 that the ideal generated by the atoms is join-dense.

7. The type III case. Let $(L, \sim)$ be a type III dimension lattice, and let $E$ be the set of invariant elements. If $0<x \in L$ and if $x$ were m.f. with respect to $E$, then $[0,|x|]$ would be a type I direct summand of $L$, so $L$ has no such elements $x$. The following theorem is the converse of this fact.

Theorem 7.1. Let $L$ be a complete oc.w.m. lattice and let $E$ be a closed Boolean subalgebra of the center. Suppose $L$ has no element $x>0$ which is $m$.f. with respect to $E$, and define $x \sim y$ to mean $|x|=|y|$. Then $(L, \sim)$ is a type III dimension lattice in which $E$ is exactly the set of invariant elements.

Proof. Axiom $\mathrm{A}$ is true because $|x| \geqq x$, and axiom $\mathrm{C}$ is true because $|\sup T|=\sup \{|t|: t \in T\}$. Axiom $\mathrm{D}^{\prime}$ is just Lemma 2.2. If $0<x \in L$, then $x$ is not m.f., so there is a $y \leqq x$ such that the element $e=|y| \wedge|x-y|$ $>0$. Then $e=|e \wedge y|=|e \wedge(x-y)|$, so every nonzero element dominates orthogonal nonzero elements with the same $E$-cover. An easy Zorn's lemma argument shows that if $0<x \in L$, then there exist orthogonal elements $y, z$ with $x=y+z$ and $|x|=|y|=|z|$. An easy application of this proves axiom $\mathrm{B}$, and it also shows that $(L, \sim)$ is type III.

Now suppose $e \in E$ and $x \prec e$. Then $|x| \leqq e$, so $x \leqq e$. Thus every element of $E$ is invariant. If $x$ is not in $E$ then $|x| \sim x$ but $|x|>x$, so $x$ is not invariant.

CoRollary 7.2. If $L$ is a complete oc.w.m. lattice with no atoms, then there is a d.e.r. on $L$ such that $(L, \sim)$ is a type III factor.

Proof. Take $E=\{0,1\}$ in Theorem 7.1.

8. The general case. Let $L$ be a complete oc.w.m. lattice and consider again the decomposition $L=L_{1} \times L_{2} \times L_{3} \times L_{4}$ of $\$ 3$.

Lemмa 8.1. There is a d.e.r. $\sim$ on $L$ if and only if $L_{4}=\{0\}$. Furthermore, $\sim$ can be chosen so that the center is the set of invariant elements if and only if $L_{3}=\{0\}$ as well.

Proof. Every atom in a dimension lattice belongs to the type I part, which is locally modular, so if a d.e.r. exists then $L_{4}=\{0\}$. A product of dimension lattices is a dimension lattice, there is a d.e.r. on $L_{1}$ by Theorem 
4.23, and there is a d.e.r. on $L_{2} \times L_{3}$ by Theorem 7.1 and Corollary 7.2, so if $L_{4}=\{0\}$ there exists a d.e.r. on $L$. If $L_{3}=\{0\}$ also, then Theorems 4.26 and 7.1 imply that $\sim$ can be chosen so that every central element is invariant. On the other hand, suppose $(L, \sim)$ is a dimension lattice with every central element invariant. Then every nonzero m.f. element is simple with respect to $\sim$ and hence belongs to the type I part of $L$, which is contained in $L_{1}$. Hence $L_{3}=\{0\}$.

TheOREM 8.2. Let $L$ be a complete oc.w.m. lattice. There exists a d.e.r. on $L$ if and only, if $(\mathrm{p})$ holds in $L$ and there exists a d.e.r. on $L$ for which every central element is invariant if and only if $\left(\mathrm{p}^{+}\right)$holds in $L$.

Proof. Straightforward applications of Theorems 6.3 and 6.7 show that $L_{4}=\{0\}$ if and only if (p) holds in $L$ and that $L_{3} \times L_{4}=\{0\}$ if and only if $\left(\mathrm{p}^{+}\right)$holds in $L$.

COROLLARY 8.3. Let $L$ be a complete oc.w.m. lattice such that if $x$ and $y$ are perspective there is an automorphism $\alpha$ of $L$ such that $\alpha(x)=y$. Then there is a d.e.r. on $L$ for which the set of invariant elements is just the center of $L$.

Proof. The multiplicity freeness of an element is preserved by automorphisms, and $x-x \wedge y$ is perspective to $x \vee y-y$, so $\left(\mathrm{p}^{+}\right)$holds in $L$.

9. Two examples. We exhibit here two examples showing that both $L_{3}$ and $L_{4}$ of $\$ \S 3$ and 8 can differ from $\{0\}$.

Let $K$ be the horizontal sum [12] of the Boolean algebra of subsets of a three element set with itself. The diagram of $K$ is in Figure 1. (The example of Dilworth [3, p. 21] would also serve as well.)

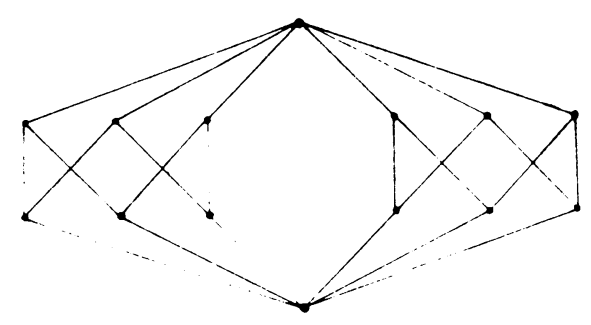

Figure 1

Then $K$ is an atomic orthomodular lattice of finite length which is not modular, and hence $K$ is an example of nonzero $L_{4}$.

Let $E$ be any complete Boolean algebra with no atoms, e.g., the Boolean algebra of Lebesgue measurable subsets of the unit interval modulo null sets. Then let $S$ be the Stone space of $E$ and let $M$ be the set of continuous functions from $S$ to the $K$ of the preceding example, $K$ having the discrete topology; $M$ is a subset of the complete orthomodular lattice of all functions from $S$ to $K, F(S ; K)$. It is easy to see that $M$ is an orthomodular 
sublattice of $F(S ; K)$, because an element of $F(S ; K)$ is continuous if and only if the inverse image of each point is open. Let $\left\{f_{i}: i \in I\right\}$ be any subset of $M$ and let $g$ be its least upper bound in $F(S ; K)$. Using the fact that the closure of an open set in $S$ is open $[17$, p. 186] and the fact that $K$ is finite, one can construct a continuous $h: S \rightarrow K$ which agrees with $g$ on an open everywhere dense set. Then $h$ is the least upper bound of $\left\{f_{i}: i \in I\right\}$ in $M$, so $M$ is complete. If $a$ is an atom in $K$ and $f$ is the constant function whose value is $a$, then it is not hard to see that $f$ is multiplicity free with respect to the natural image of $E$ in $M$. Thus $M$ is complete and orthomodular and has a multiplicity free element with central cover 1 , but $M$ has no locally modular direct summand. Hence $M$ gives an example of a nonzero $L_{3}$.

\section{REFERENCES}

1. I. Amemiya and I. Halperin, Complemented modular lattices, Canad. J. Math. 11 (1959), 481-520.

2. G. Birkhoff, Lattice theory, Amer. Math. Soc. Colloq. Publ. Vol. 25, Amer. Math. Soc., Providence, R. I., 1948.

3. R. P. Dilworth, On complemented lattices, Tôhoku Math. J. 47 (1940), 18-23.

4. J. Dixmier, Sur certains espaces considérés par M. H. Stone, Summa Brasil. Math. 2 (1951), 151-182.

5. D. J. Foulis, Conditions for the modularity of an orthomodular lattice, Pacific J. Math. 11 (1961), 889-895.

6. S. S. Holland, Jr., Distributivity and perspectivity in orthomodular lattices, Trans. Amer. Math. Soc. 112 (1964), 330-343.

7. T. Iwamura, On continuous geometries. I, Japan. J. Math. 9 (1944), 57-71.

8. I. Kaplansky, Any complete orthocomplemented modular lattice is a continuous geometry, Ann. of Math. (2) 61 (1955), 524-541.

9. L. H. Loomis, The lattice theoretic background of the dimension theory of operator algebras, Mem. Amer. Math. Soc. No. 18 (1955), 36 pp.

10. G. W. Mackey, On infinite-dimensional linear spaces, Trans. Amer. Math. Soc. 57 (1945), 155-207.

11. Infinite-dimensional group representations, Bull. Amer. Math. Soc. 69 (1963), 628-686.

12. M. D. MacLaren, Horizontal sums of orthocomplemented lattices, Notices Amer. Math. Soc. 10 (1963), 63.

13. Atomic orthocomplemented lattices, Math. Note No. 286, Boeing Scientific Research Laboratories, Seattle, Washington, 1963.

14. F. Maeda, Kontinuierliche Geometrien, Springer-Verlag, Berlin, 1958.

15. S. Maeda, Dimension functions on certain general lattices, J. Sci. Hiroshima Univ. Ser. A-I Math. 19 (1955), 211-237.

16. M. H. Stone, Boundedness properties in function-lattices, Canad. J. Math. 1 (1949), 176-186.

17. The theory of representations for Boolean algebras, Trans. Amer. Math. Soc. 40 (1936), 37-111.

18. J. von Neumann, C'ontinuous geometry, Princeton Univ. Press, Princeton, N. J., 1960.

HaRVARD UNIVERSITY,

Cambridge, Massachusetts

Massachusetts Institute of Technology, Cambridge, Massachusetts 